\title{
THE EQUATION OF STATE OF MATTER AT SUB-NUCLEAR DENSITY
}

\author{
J. W. NEGELE \\ Laboratory for Nuclear Science and Department of Physics, \\ Massachusetts Institute of Technology, Cambridge, Mass. 02139, U.S.A.
}

\begin{abstract}
An extremely simple form for the energy density of a nuclear many-body system is derived from the two-body nucleon-nucleon interaction. This theory, which yields excellent results for energies and density distributions of finite nuclei, is used to determine the ground state configuration of matter at sub-nuclear density. As the baryon density is increased, nuclei become progressively more neutron rich until neutrons eventually escape, yielding a Coulomb lattice of bound neutron and proton clusters surrounded by a dilute neutron gas. The clusters enlarge and the lattice constant decreases with increasing density, approaching a completely uniform state near nuclear density.
\end{abstract}

\section{Introduction}

There exists sufficient astrophysical evidence that pulsars are in fact neutron stars that it is worthwhile to seriously investigate the equation of state of dense baryonic matter utilizing one's current understanding of theoretical nuclear physics. In the present work, we shall restrict our attention to that limited regime in which the two tacit assumptions of nuclear physics are satisfied, namely that the temperature be low compared with characteristic nuclear excitation energies and that the meson degrees of freedom may be legitimately suppressed and replaced by a phenomenological two-body potential determined by scattering data and deuteron properties.

The first condition appears to be satisfied for observed pulsars, since the surface temperature may be inferred to be less than $10^{8} \mathrm{~K}$, corresponding to $10 \mathrm{keV}$ and the thermal conductivity is expected to be very high. Thus, if we ignore the early stages of formation, on the relevant temperature scale of nuclear energies, neutron stars are exceedingly cold and may be treated in first approximation as being at a temperature of absolute zero. Hence it is legitimate to use the same zero-temperature perturbation expansions which are used in nuclear many-body theory, and the equation of state is completely specified by calculating the binding energy per baryon as a function of baryon density.

The suppression of meson degrees of freedom in nuclear physics has been extremely successful. At a density of 0.17 nucleons $\mathrm{fm}^{-3}$, corresponding to the interior density of a heavy nucleus, the average distance between nuclei is $2.2 \mathrm{fm}$, whereas the one pion exchange force has a range of $1.4 \mathrm{fm}$. Thus, it appears quite reasonable to first approximate the interaction between two nucleons in nuclear matter as the interaction which would occur in free space, and then treat as a small perturbation the explicit meson many-body effects. The lowest order example of such an effect would be the three-body force arising from the interaction of a pion in transit between two nucleons with yet a third nucleon. Such processes have been investigated in nuclear matter and yield a correction to the binding energy per particle on the order of $2 \mathrm{MeV}$ out of a 
total potential energy contribution of $40 \mathrm{MeV}$ (Brown and Green, 1969). Thus, present evidence indicates that if we restrict our attention to nuclear configurations with densities less than or equal to that occurring in the interior of large nuclei, the structure should be dominated by the two-body nucleon-nucleon interaction and explicit meson many-body effects should produce only very small corrections amenable to perturbation theory.

Having determined the upper limit on densities to be considered to be that occurring in the interior of large nuclei, hereafter referred to simply as nuclear density and having the value 0.17 nucleon $\mathrm{fm}^{-3}=2.8 \times 10^{14} \mathrm{~g} \mathrm{~cm}^{-3}$, we now consider the lower limit.

At densities above $10^{7} \mathrm{~g} \mathrm{~cm}^{-3}$ for temperatures below $10^{8} \mathrm{~K}$, matter is expected to be a solid. This is because the Coulomb interaction between ions is only weakly screened and at sufficiently low temperatures, the Coulomb energy is minimized by a bcc lattice. Applying the Lindemann melting criterion which requires the mean fluctuations of ions to be small compared with the average ion spacing, the ions are expected to form a solid below the temperature $T_{m} \approx Z^{2} e^{2} /\left(100 r_{Z}\right)$ where $r_{Z}$ is the average spacing between ions (Pines, 1970). For ${ }^{56} \mathrm{Fe}$, this solidification occurs by the time one reaches a density of $10^{7} \mathrm{~g} \mathrm{~cm}^{-3}$, and as the density increases, $r_{Z}$ decreases, assuring that the condition will be maintained at all subsequent densities. At extremely high densities, one might worry the Coulomb lattice breaking up solely due to the zero point motion of the ions, but for the highest density configuration treated in this work, ${ }_{32} \mathrm{Ge}$ at $1.4 \times 10^{14} \mathrm{~g} \mathrm{~cm}^{-3}$, the zero point fluctuation is still very small compared with the ion spacing.

For Coulomb lattices of ions at densities between $10^{7}$ and $10^{9} \mathrm{~g} \mathrm{~cm}^{-3}$, the equation of state is determined directly from the experimentally observed mass table using straightforward corrections for the electrons and lattice Coulomb energy (Baym et al., 1971a). At $\varrho=6.2 \times 10^{9} \mathrm{~g} \mathrm{~cm}^{-3}$, the Fermi energy of the relativistic electrons shifts the energy balance favoring neutron rich nuclei so strongly that ${ }^{84} \mathrm{Se}$, which is unstable but experimentally observable, gives way to ${ }^{82} \mathrm{Ge}$ which has not been observed but may be reliably extrapolated (Myers and Swiatecki, 1965). Extrapolations from the mass table continue to be reliable up to a density of roughly $4.3 \times 10^{11} \mathrm{~g} \mathrm{~cm}^{-3}$, at which point the neutrons in ${ }^{118} \mathrm{Kr}$ are just barely bound and any further increase in density causes the last few neutrons to 'drip' out of the nuclei and form a low density neutron gas in the intervening space between the nuclear clusters. Although the theory presented in the present work should also be valid in the entire pre-drip sequence of extrapolated nuclei, we believe the mass table extrapolations are satisfactory well below the drip point and have concentrated our attention on the last sequence of pre-drip nuclei with 82 neutrons: ${ }^{124} \mathrm{Mo},{ }^{122} \mathrm{Zr},{ }^{120} \mathrm{Sr}$ and ${ }^{118} \mathrm{Kr}$, and on the free neutron regime after drip occurs, between $4 \times 10^{11} \mathrm{~g} \mathrm{~cm}^{-3}$ and $2 \times 10^{14} \mathrm{~g} \mathrm{~cm}^{-3}$.

In order to assess the significance of this region of densities, calculations of stellar density distributions by Baym et al. (1971a) using an equation of state by Baym et al. (1971b) which is very similar to that obtained in this work, are shown in Figure 1. From this graph, it is observed that the very lightest neutron stars do not exceed nuclear density and that the region from $4 \times 10^{11}$ to $2 \times 10^{14} \mathrm{~g} \mathrm{~cm}^{-3}$ is the most crucial 
in determining the structure and stability of these stars. In particular, one may observe qualitatively the effect of neutron drip and the approach to nuclear density on the equation of state. At $4 \times 10^{11} \mathrm{~g} \mathrm{~cm}^{-3}$ where neutron drip occurs, the sudden increase in slope of the density distribution indicates the appreciable softening of the equation of state when neutrons begin to drip out of nuclei. Similarly the flatness of the curve near nuclear density reflects the stiffness of the equation of state once nuclei begin to touch and the gravitational pressure attempts to compress the nucleons to a density

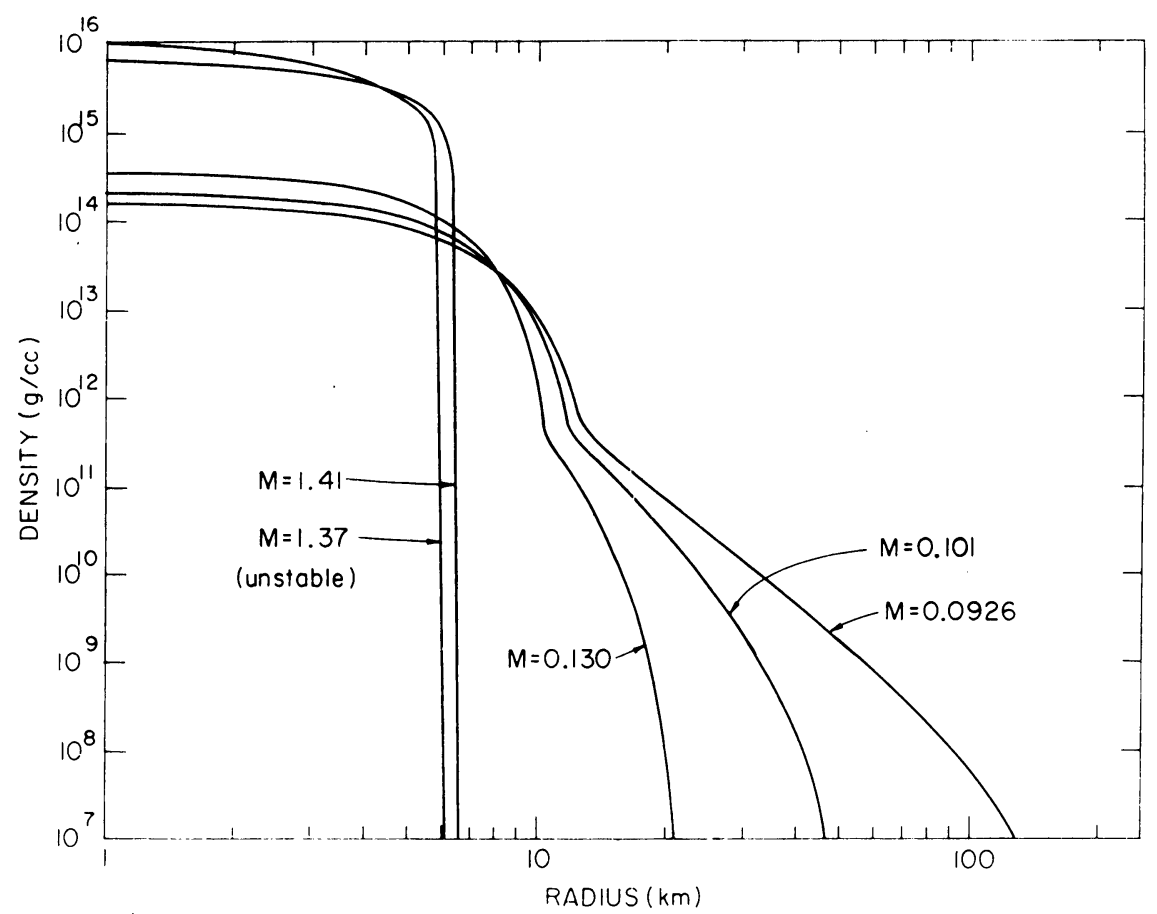

Fig. 1. Density profiles of neutron stars, calculated by Baym et al. (1971a).

higher than that occurring in a free nucleus. The need for quantitative precision in describing both the softness at the neutron drip point and stiffness near nuclear density motivates the present application of a microscopic theory of nuclear structure to the examination in somewhat greater detail of the equation of state in this regime.

\section{Review of Previous Investigations}

It is natural that the first attempts to treat the free neutron regime should be based on extrapolations from the semi-empirical mass formula (Bethe et al., 1970; Langer et al., 1969). The limitations of such an approach arise, obviously, from the fact that the mass formula parameters are determined only by a very restricted region of nuclear 
configurations: the ratio of protons to neutrons is greater than $\sim 0.6$, the chemical potential (minus the removal energy) for neutrons and protons is on the order of $-8 \mathrm{MeV}$ and there is zero external pressure on the nucleus. In the free neutron regime, however, the configurations are very far from satisfying these conditions. The ratio of protons to neutrons in nuclei is 0.1 to 0.3 ; the neutron chemical potential in the nucleus must equal that of the free gas and thus cover a range from 0 to $+20 \mathrm{MeV}$; the pressure from the exterior neutron gas becomes significant, and the surface becomes much more diffuse and instead of approaching zero density outside the nucleus, approaches the neutron gas density. For all these reasons, extrapolations based on the semiempirical mass formula are extremely unreliable, and one is forced to undertake a more fundamental theory.

Baym et al. (1971b, hereafter denoted BBP) introduced the information which is unobtainable from the mass formula by means of the theory of uniform nuclear matter. Based on nuclear matter calculations with the Reid (1968) soft core potential by Siemens (1970) in the region of roughly equal neutron and proton densities and by Siemens and Pandharipande (1971) in the region of almost pure neutron matter, BBP obtained the nuclear binding energy per nucleon surface shown in Figure 2. One should note that both Coulomb and gravitational interactions are necessarily omitted from these infinite nuclear matter calculations. In addition, since only two-body reaction matrix diagrams are included, a phenomenological correction was applied

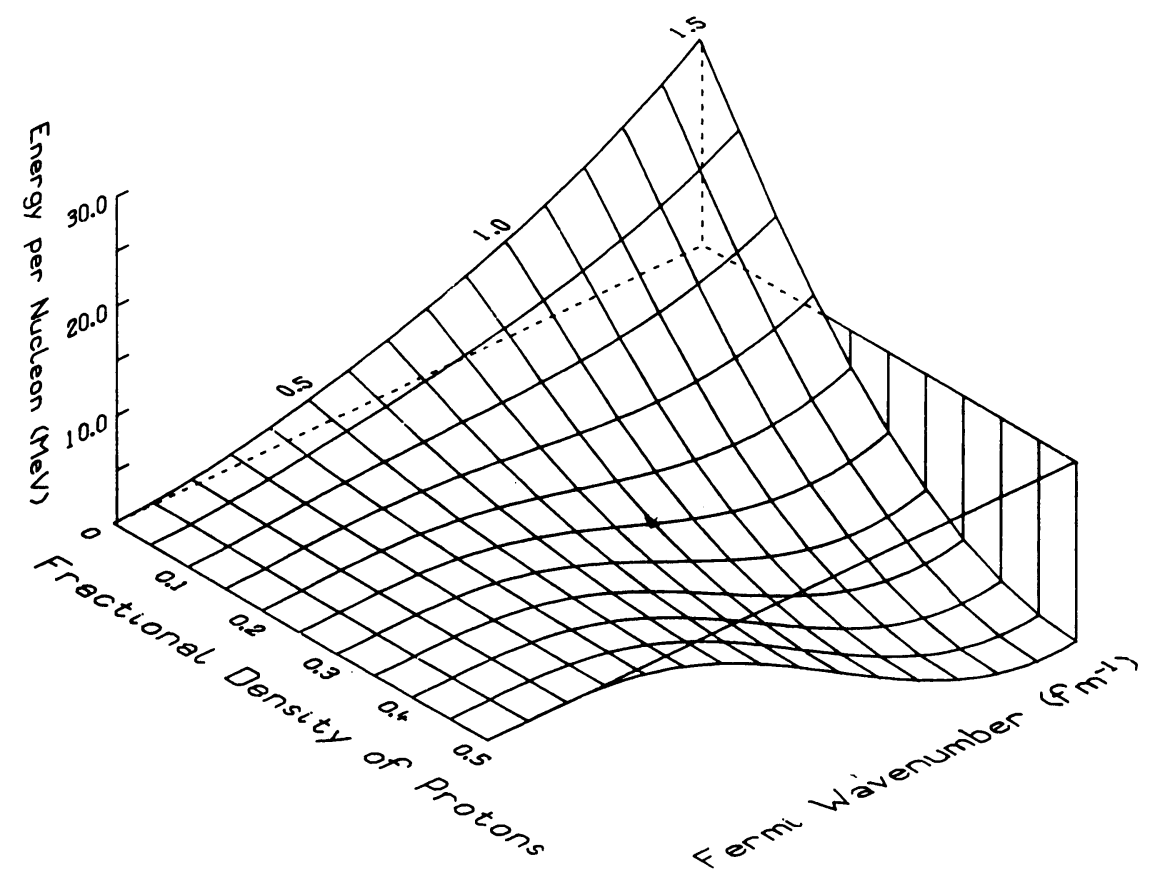

Fig. 2. Energy per nucleon of uniform nuclear matter ignoring Coulomb and gravitational forces, taken from BBP. 
to include the effects of all higher order diagrams, three-body forces, and relativistic corrections. Since such corrections enter most strongly in the ${ }^{3} S_{1}-{ }^{3} D_{1}$ coupled partial waves which are connected by the tensor force, the phenomenological correction was defined to occur only in isospin $T=0$ states and thus acts only between unlike particles. By means of this correction, the minimum of the saturation curve was shifted from $11 \mathrm{MeV}$ to $16.5 \mathrm{MeV}$ at a density corresponding to $k_{\mathrm{F}}=1.43 \mathrm{fm}^{-1}$.

Having determined the binding energy surface for all densities and ratios of protons and neutrons, BBP treat the Coulomb lattice of nuclei surrounded by a neutron gas in the Wigner-Seitz approximation. The unit cell is divided into a uniform density interior nucleus comprised of neutrons at high density and protons of low density and a uniform neutron gas exterior, comprised of neutrons at low density. A phenomenological surface energy expression motivated by Thomas-Fermi theory corrects for the presence of a finite surface and the Coulomb energy is calculated assuming a constant density of electrons filling the entire cell to yield charge neutrality. Then at a specified baryon density, the ground state configuration is determined by requiring that the system be stable against $\beta$-decay, that the pressure inside the nucleus equal the pressure in the outside gas, that the neutron chemical potential inside the nucleus equal that of the outside gas and by minimizing the total binding energy with respect to the radius of the unit cell.

Whereas this approach constitutes a clear improvement over previous work, it is still subject to two limitations. The first is an incomplete theory of the nuclear surface, with the general form being specified by Thomas-Fermi theory, but with the actual surface thickness being determined from the neutron Fermi wavelength instead of variationally. The second is the complete absence of nuclear shell effects, which are already observed to play an important role in determining the composition of the ground state matter prior to neutron drip.

Similar calculations by Arponen (1971), Barkat et al. (1972) and Buchler and Barkat $(1971 \mathrm{a}, \mathrm{b})$ utilize slightly different parameterizations of the nuclear energy surface and determine ground state density distributions variationally in a Thomas-Fermi theory. The surface energy is included by introducing a gradient term in the expression for the energy as a functional of the density with a phenomenological coefficient adjusted to fit ordinary nuclei.

The semi-classical Thomas-Fermi approximation has been compared with the quantum mechanical Hartree-Fock method for a semi-infinite surface by Ravenhall et al. (1972). Using the Skyrme (1959) interaction, they demonstrate significant differences between the density distributions and equilibrium configurations with the two methods (see also Vautherin and Brink, 1972). The final result they obtain in Hartree-Fock theory for the charge of the nucleus as a function of matter density lies between the results of BBP and Barkat et al. (1972) and constitutes the most reliable calculation discussed thus far. We will subsequently compare the calculations of this present work with those of Ravenhall et al. (1972) and show how the differences arise from curvature terms in the surface energy and nuclear shell effects, both of which are necessarily omitted in a semi-infinite matter calculation. 


\section{Theory of Finite Nuclei}

Present evidence indicates that a strongly repulsive core in the nucleon-nucleon interaction plays an essential role in producing finite nuclei which obtain the proper binding energy without collapsing to unphysically high densities. Once one adopts a potential with a strongly repulsive core, such as the Reid soft core potential used in this work, perturbation theory can no longer be ordered simply in terms of numbers of interactions of the bare potential, but rather must be re-expressed in terms of the reaction matrix. The reaction matrix, $G$, sums all orders of ladder diagrams containing the bare interaction and unoccupied intermediate states and may be written

$$
G(w)=v-v \sum_{a b} \frac{|a b\rangle\langle a b|}{e_{a}+e_{b}-W} G(w),
$$

where the projector labels $a, b$ are summed over all unoccupied states and $W$ indicates an energy specified by the single particle energies of the two interacting particles and the particular Goldstone diagram under consideration.

Physically, by permitting two interacting particles to interact any number of times when they approach each other, one is allowing the two-body wave function to respond to the presence of the potential by generating correlations which strongly decrease the probability of the particles penetrating into the repulsive core region. This may be visualized by defining the correlated wave function $\psi$ by the relation $G \phi \equiv v \psi$, where $\phi$ is the uncorrelated wave function which is a plane wave in nuclear matter. The correlated wave function in the ${ }^{1} S_{0}$ partial wave channel obtained in this way in nuclear matter is shown in Figure 3.

The field of nuclear matter theory is treated in great detail in two recent extensive review articles by Bethe (1971) and Sprung (1972). For our present purposes, however, it is sufficient to concentrate our attention on the general feature of the two-body correlations. The difference between $\phi$ and $\psi$, which we shall refer to as the defect function and denote by $\chi$, contains all the information concerning two-body correlations and will play a central role in our theory of finite nuclei.

Whereas Figure 3 only shows the defect function in a single partial wave, one can more effectively visualize the total two-body correlation by plotting the sum of the squares of the defect functions in each partial wave as shown in Figure 4 for several densities of nuclear matter. The integral of the sum of the squares of the defect functions, multiplied by the density and appropriate statistical factors, yields the total probability of exciting a particle out of the uncorrelated Fermi sea into some excited state, which turns out to be approximately $13 \%$ at nuclear density. The most striking feature displayed in Figure 4 is that in addition to the maximum at $0.5 \mathrm{fm}$ expected from the fact that the correlated wave function does not penetrate the hard core significantly, there is a second maximum at $1.2 \mathrm{fm}$. This correlation arises from second and higher order processes in which the strong, long range tensor force coupling the ${ }^{3} S_{1}-{ }^{3} D_{1}$ channels introduces deuteron-like spatial correlations in the two-body wave 
function. A particularly significant feature of this correlation is its strong density dependence. Recalling the definition of the reaction matrix, it is clear that at high density there are fewer intermediate states available for second order contributions to $\psi$, and Figure 4 shows how strongly these correlations are diminished at high density.

Having investigated the two-body correlations occurring between nucleons in nuclear matter, we now apply this knowledge to finite nuclei via the local density approximation. Physically, one argues that the short range correlations between two nucleons in the interior of ${ }^{208} \mathrm{~Pb}$, which is roughly $13 \mathrm{fm}$ in diameter, should be the same as for two nucleons in infinite uniform matter at the same neutron and proton density. For nucleons near the nucleon surface, which is $2 \mathrm{fm}$ thick, the argument is still quite good for the $0.4 \mathrm{fm}$ correlations due to the core, and begins to break down only for the longer range tensor force correlations.

The most convenient technique for using the correlations calculated in nuclear matter in finite nuclei is the construction of an effective interaction. Considering, for simplicity, relative matrix elements in a finite nucleus in a specific relative partial wave one may introduce a complete set of plane waves and define a non-local effective interaction as follows:

$$
\begin{aligned}
\left\langle\phi_{n l}|G| \phi_{n l}\right\rangle & =\sum_{k}\left\langle\phi_{n l}|G| \phi_{k}\right\rangle\left\langle\phi_{k} \mid \phi_{n^{\prime} l}\right\rangle= \\
& =\iint\left\langle\phi_{n l} \mid r\right\rangle\left\langle r\left|\sum_{k} v\right| \Psi_{k}\right\rangle\left\langle\phi_{k} \mid r^{\prime}\right\rangle\left\langle r^{\prime} \mid \phi_{n^{\prime} l}\right\rangle \mathrm{d}^{3} r \mathrm{~d}^{3} r^{\prime} \equiv \\
& \equiv \iint \phi_{n l}(r) v_{\text {eff }}\left(\varrho, W, r, r^{\prime}\right) \phi_{n^{\prime} l}\left(r^{\prime}\right) \mathrm{d}^{3} r \mathrm{~d}^{3} r^{\prime}
\end{aligned}
$$

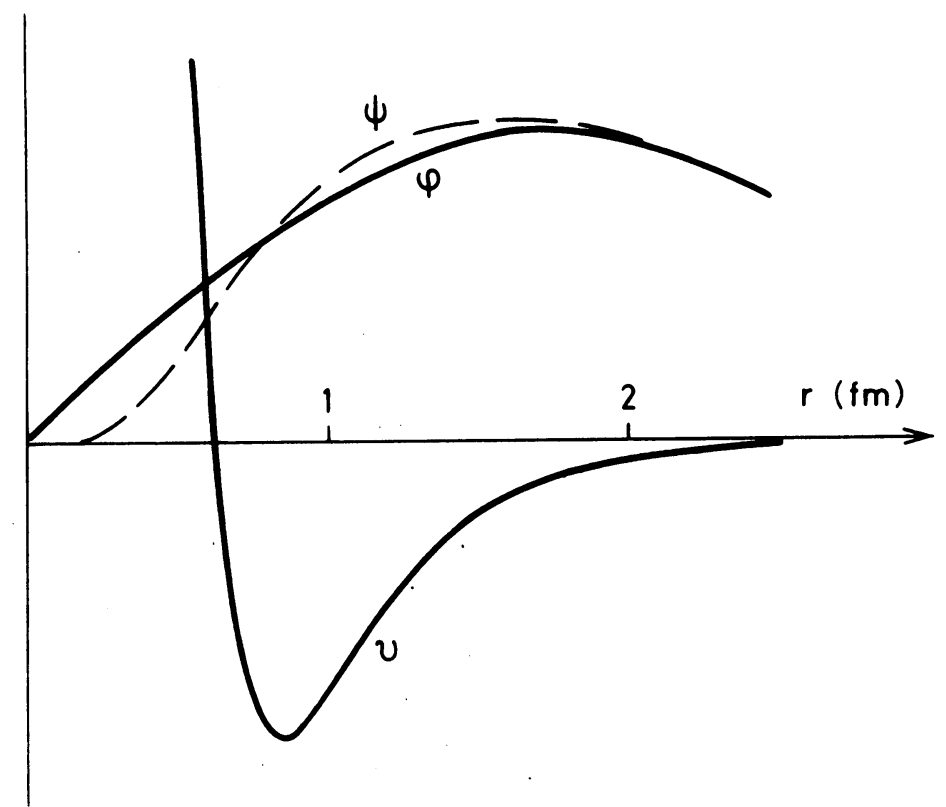

Fig. 3. Reid potential and uncorrelated and correlated nuclear matter wave functions for the ${ }^{1} S_{0}$ partial wave. 
For a practical calculation, it is necessary to make a number of additional simplifying approximations to $v_{\text {eff }}$ yielding an effective interaction which is local in relative coordinate and is averaged over relative angular momentum states in each spin and isospin channel. These approximations are justified in detail elsewhere (Negele, 1970) and are immaterial to the conceptual basis of the theory. The final result is an effective interaction to be used with a Slater determinant wave function which depends not only on the relative coordinate of two interacting particles, but also on the density at the location where the two particles are interacting and on the energy of the two interacting particles. The fact that both the density dependence and energy dependence

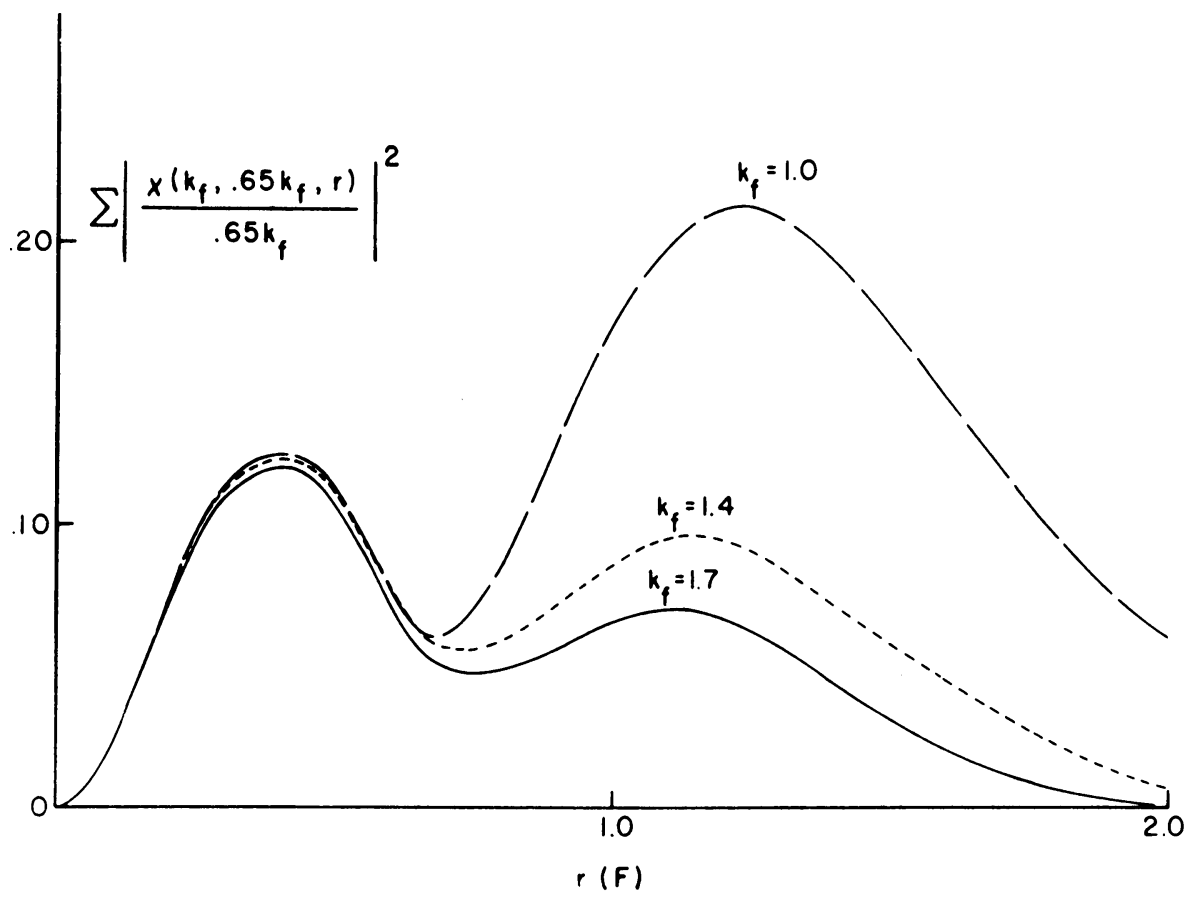

Fig. 4. Sum of the squares of the defect functions in nuclear matter taken from Negele (1970).

contribute to saturation, that is to making the interaction less attractive at high density, is evident from examining the second order term in the reaction matrix expansion, $v(Q / e) v$. As the density increases, the projector onto unoccupied states, $Q$, excludes more and more phase space from the second order sum, which is always attractive, thereby decreasing the attraction. In addition, the single particle energies become larger in magnitude with increasing density, thereby increasing the magnitude of the energy denominator $e$ and decreasing the attraction.

Once one has accepted an effective interaction which is density and energy dependent, it is straightforward to write out the expression for the energy of a Slater determinant and to functionally differentiate with respect to each single particle wave function. In addition to the usual Hartree-Fock terms obtained from varying $\psi_{m}^{*}\left(r_{1}\right)$ 
and $\psi_{n}^{*}\left(r_{2}\right)$ appearing explicitly in

$$
\begin{aligned}
& \sum_{m n} \iint \Psi_{m}^{*}\left(r_{1}\right) \Psi_{n}^{*}\left(r_{2}\right) g\left[\left|r_{1}-r_{2}\right|\right.\left.\varrho\left(\frac{r_{1}-r_{2}}{2}\right), e_{m}+e_{n}\right] \times \\
& \times\left[\Psi_{m}\left(r_{1}\right) \Psi_{n}\left(r_{2}\right)-\Psi_{n}\left(r_{1}\right) \Psi_{m}\left(r_{2}\right)\right]
\end{aligned}
$$

one also obtains terms of the form $(\partial G / \partial \varrho)\left(\partial \varrho / \partial \psi_{s}^{*}\right)$ and $\left(\partial G / \partial e_{m}\right)\left(\partial e_{m} / \partial \psi_{s}^{*}\right)$. These terms express the fact that by increasing the probability of finding particle $s$ at some region in the nucleus, the total energy contribution from that region is not only increased because of the pair interactions of particle $s$ with all other nucleons, but it is also slightly decreased by the fact that two other particles, say $m$ and $n$ interacting in that region, now find their interactions less attractive due to the fact that particle $s$ has now made some phase space unavailable for intermediate scattering states and has slightly increased their energy denominators. These extra terms in the resulting densitydependent Hartree-Fock (DDHF) equations, loosely referred to as 'rearrangement terms' are crucial to obtaining saturation in finite nuclei and in obtaining agreement with experimental single particle energies and binding energies.

The effective interaction described above is actually defined in detail to reproduce exactly the same nuclear matter saturation curve as Siemens' (1970) original $G$ matrix when evaluated in a Fermi gas of plane wave states. Thus, in order to obtain the proper binding energy per particle, it is necessary to introduce a phenomenological correction to include all the higher order terms in nuclear matter theory. In the same spirit as BBP, this was chosen to be a short range force acting only between unlike particles and for the results presented in this work, the two parameters were adjusted to give $16.53 \mathrm{MeV}$ binding energy per particle at a saturation density of $k_{\mathrm{F}}=1.33 \mathrm{fm}^{-1}$.

For spherical nuclei, it is straightforward, although numerically cumbersome, to directly solve the non-local integro-differential DDHF equations and by iteration obtain self-consistent wave functions. The binding energies per particle obtained in this way (Bethe, 1971) agree with experimental energies for $\mathrm{O}, \mathrm{Ca}, \mathrm{Zr}$ and $\mathrm{Pb}$ to within $0.5 \mathrm{MeV}$, and subsequent calculations are in even closer agreement (Negele, 1970) and (Campi and Sprung, 1972). The single particle energies agree with experimental energies to within several $\mathrm{MeV}$ and in particular the spin-orbit splittings in light nuclei are correct.

Having shown the necessity of having an accurate quantum mechanical theory of the nuclear surface, we wish to emphasize the agreement of the DDHF results with experimental evidence concerning the surface. The agreement of binding energies throughout the periodic table mentioned previously indicates the semi-empirical surface energy parameter is accurately reproduced. In addition, it is possible to check the detailed spatial distribution of protons by comparison with elastic electron scatterings results, as shown in Figures 5 and 6 for ${ }^{40} \mathrm{Ca}$ and ${ }^{208} \mathrm{~Pb}$, respectively. Although an accurate fit for a single nucleus might be deemed fortuitous, systematic agreement throughout the periodic table yields strong evidence that the delicate balance between Coulomb energy, symmetry energy, surface energy and bulk volume energy is being very accurately reproduced. 
The DDHF theory of finite nuclei as described above is unfortunately too computationally time-consuming to apply to the equation of state in the free neutron regime. Self-consistent calculations for 208 particles in ${ }^{208} \mathrm{~Pb}$ are already lengthy and expensive and in the free neutron regime, one desires to search over a variety of configurations with up to 5000 particles in a unit cell.

The primary computational complication in the DDHF theory is the presence of the non-local exchange term, which necessitates both the Legendre expansion of the exchange potential and the explicit solution of a non-local Schrödinger equation. For this reason, it is useful to examine the structure of the exchange term and develop a systematic expansion which treats it in a much more convenient manner. Although

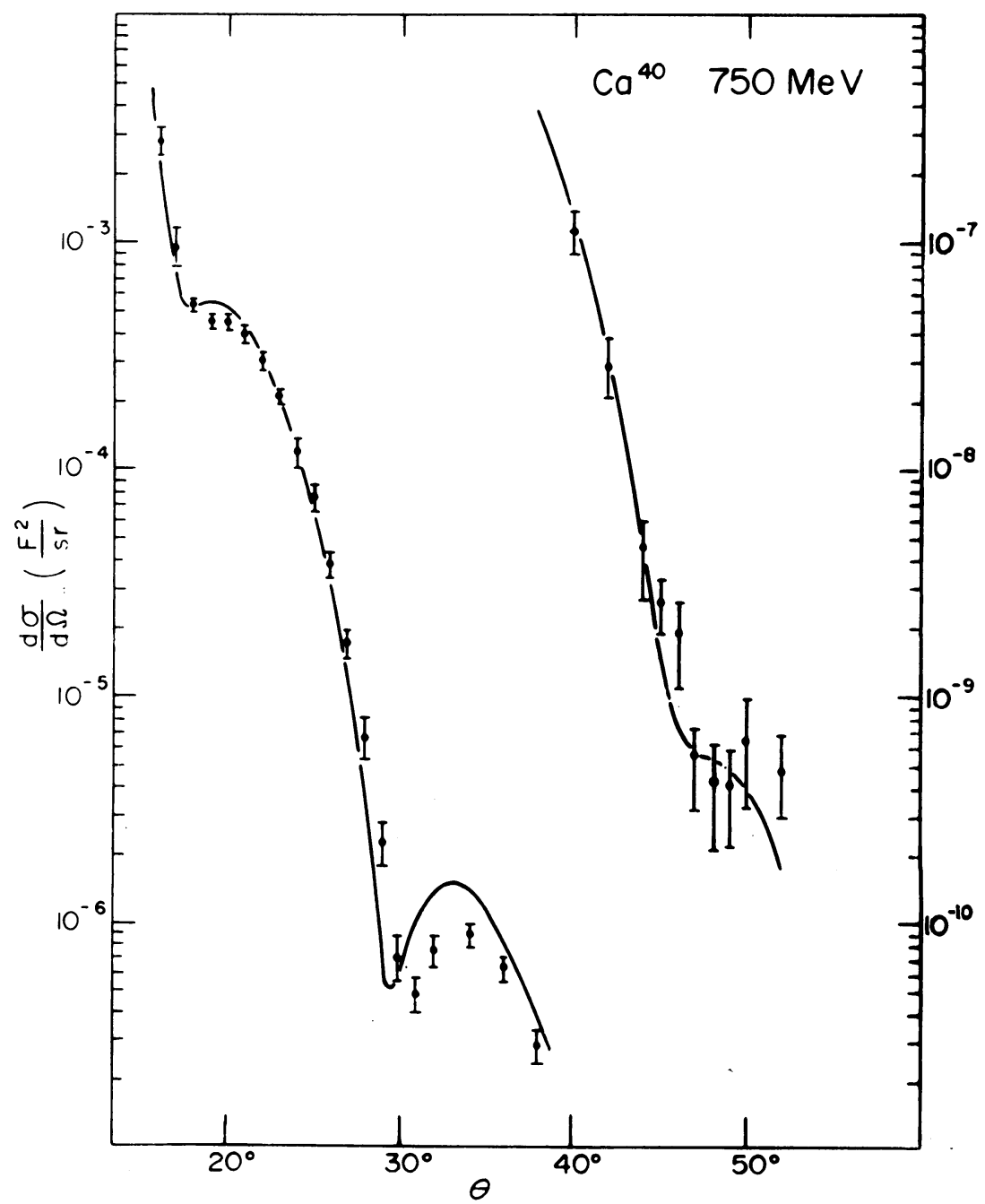

Fig. 5. Elastic electron scattering cross sections calculated with DDHF wave functions, compared with experimental results for ${ }^{40} \mathrm{Ca}$. 
this is a crucial practical development, it should be emphasized that it introduces no new conceptual assumptions since one can construct the exact exchange term and compare it with the subsequent expansion and insist on any specified degree of accuracy.

The density matrix expansion (DME) is a systematic expansion of the two-body density matrix $\varrho\left(r_{1}, r_{2}\right)=\sum_{n} \psi_{n}^{*}\left(r_{1}\right) \psi_{n}\left(r_{2}\right)$ in coordinate space (Negele and Vautherin, 1972). One begins by writing the angle-averaged density matrix as a formal expansion about the center of mass of the two interacting particles,

$$
\begin{aligned}
\hat{\varrho}(\mathbf{R}, r) & =\int \frac{\mathrm{d} \Omega}{4 \pi} \sum_{a} \psi_{a}^{*}\left(\mathbf{R}+\frac{\mathbf{r}}{2}\right) \psi_{a}\left(\mathbf{R}-\frac{\mathbf{r}}{2}\right)= \\
& \left.=\int \frac{\mathrm{d} \Omega}{4 \pi} \exp \frac{\mathbf{r} \cdot\left(\nabla_{1}-\nabla_{2}\right)}{2}\right\} \sum_{a} \psi_{a}^{*}\left(R_{1}\right) \psi_{a}\left(R_{2}\right) \\
& =\frac{1}{2} \int d \cos \theta \exp \left\{\cos \theta r\left(\frac{\nabla_{1}-\nabla_{2}}{2}\right)\right\} \varrho\left(R_{1}, R_{2}\right) \\
& \equiv F\left[\left(\frac{\nabla_{1}-\nabla_{2}}{2}\right)^{2}\right] \varrho\left(R_{1}, R_{2}\right) \\
F\left(k^{2}\right) & = \begin{cases}\frac{\sinh (k r)}{k r} & k^{2}>0 \\
\frac{\sin (k r)}{k r} & k^{2}<0 .\end{cases}
\end{aligned}
$$

Expanding this around some value $-k^{2}$, one obtains

$$
\begin{aligned}
F\left[\left(\frac{\nabla_{1}-\nabla_{2}}{2}\right)^{2}\right] & =F\left(-k^{2}\right)+F^{\prime}\left(-k^{2}\right)\left[\left(\frac{\nabla_{1}-\nabla_{2}}{2}\right)^{2}+k^{2}\right]+\cdots \\
& =j_{0}(k r)+\frac{r}{2 k} j_{1}(k r)\left[\left(\frac{\nabla_{1}-\nabla_{2}}{2}\right)^{2}+k^{2}\right]+\cdots
\end{aligned}
$$

Physically, the operator $\left(\nabla_{1}-\nabla_{2}\right) / 2$ is just the relative momentum operator. Hence it is reasonable to average both sides of this expression over the values of relative momentum $k$ which would appear in nuclear matter at the same density. Averaging with the appropriate phase space factors and suitably rearranging the series, one obtains

$$
\begin{aligned}
& \hat{\varrho}(\mathbf{R}, r)=\varrho_{\mathrm{SL}}\left(r k_{\mathrm{F}}\right) \varrho(\mathbf{R})+r^{2} g\left(r k_{\mathrm{F}}\right)\left[\frac{1}{4} \nabla^{2} \varrho(\mathbf{R})-\tau(\mathbf{R})+\frac{3}{5} k_{\mathrm{F}}^{2} \varrho(\mathbf{R})\right] \\
& \varrho_{\mathrm{SL}}\left(r k_{\mathrm{F}}\right)=\frac{3 j_{1}\left(r k_{\mathrm{F}}\right)}{r k_{\mathrm{F}}} \varrho(R)=\sum_{a}\left|\psi_{a}(\mathbf{R})\right|^{2} \\
& g\left(r k_{\mathrm{F}}\right)=\frac{35 j_{3}\left(r k_{\mathrm{F}}\right)}{2\left(r k_{\mathrm{F}}\right)} \tau(R)=\sum_{a}\left|\nabla \psi_{a}(\mathbf{R})\right|^{2} .
\end{aligned}
$$

The first term is just the Slater mixed density for a Fermi gas and the subsequent terms 
systematically expand the deviation of the finite nucleus density matrix from the nuclear matter result.

The accuracy of the first two terms is demonstrated in Figure 7. The exact density matrix is calculated for neutron wave functions in ${ }^{208} \mathrm{~Pb}$ as a function of relative coordinate, $s$, at several values of the center of mass coordinate, $R$. Since the exact density matrix is not rigorously isotropic in $\mathbf{s}$, the extremal values are indicated on the graph by the error bars. The Slater approximation, denoted by the short dashed line, is observed to be in excellent agreement with the exact calculation in the interior and to systematically overestimate the mixed density in the surface. The sum of the first two terms is indicated by the solid line and yields an excellent approximation through-

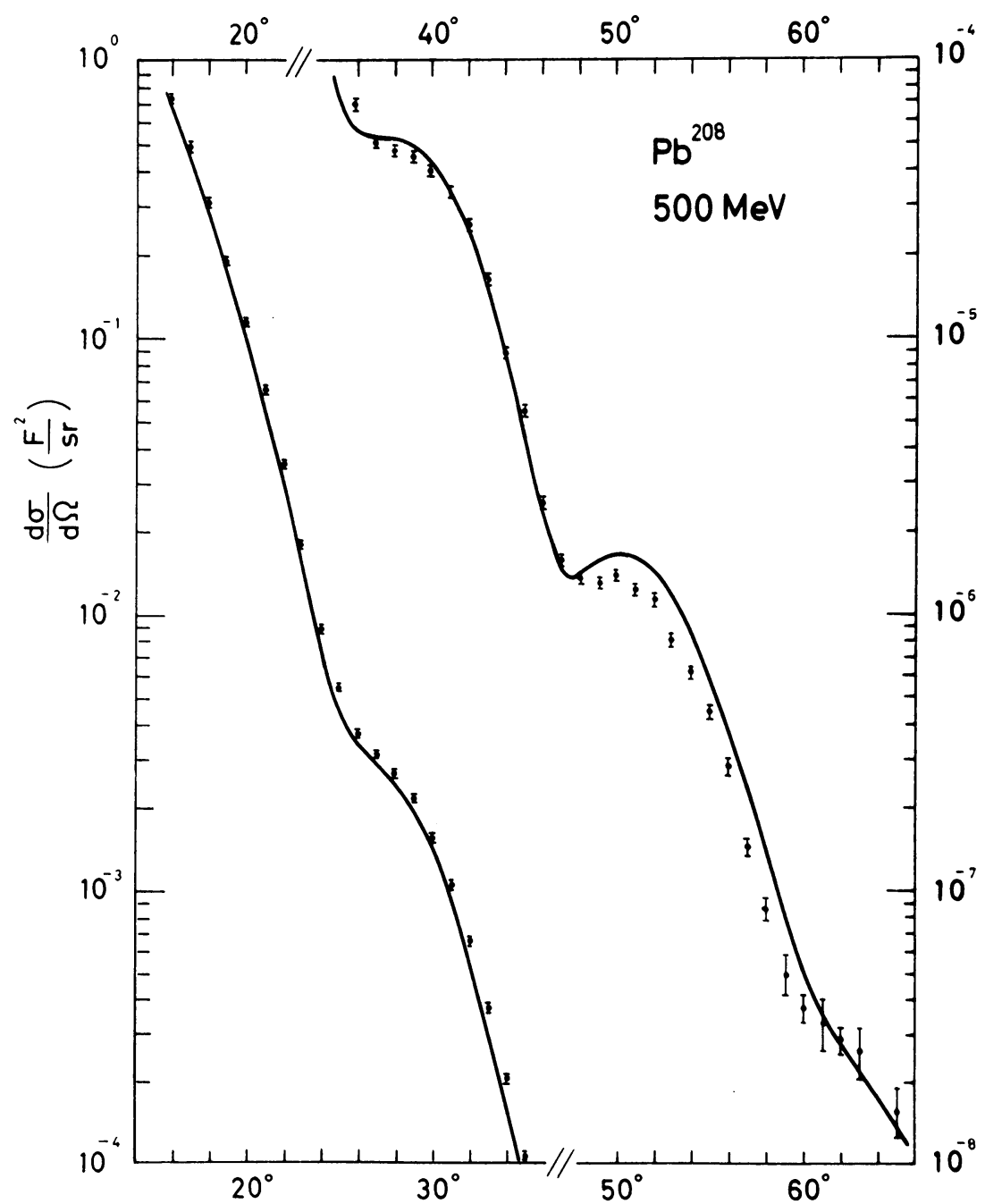

Fig. 6. Elastic electron scattering cross sections calculated with DDHF wave functions, compared with experimental results for ${ }^{208} \mathrm{~Pb}$. 
out the region of relative coordinate in which the two-body potential is significant.

Truncation of the expansion of the density matrix at second order yields an extremely simple factorized expression of specified functions of relative coordinate multiplying $\varrho(R)$, derivatives of $\varrho(R)$ and the kinetic energy density, $\tau(R)$. The total Hamiltonian density may then be obtained by performing the integral over relative coordinates of the density matrix squared times the effective interaction. Distinguishing between proton and neutron densities and kinetic energies, the final form of the Hamiltonian density is

$$
\begin{aligned}
H(R)=\frac{\hbar^{2}}{2 M}\left(\tau_{\mathrm{n}}\right. & \left.+\tau_{\mathrm{p}}\right)+A\left(\varrho_{\mathrm{n}}, \varrho_{\mathrm{p}}\right)+B\left(\varrho_{\mathrm{p}}, \varrho_{\mathrm{n}}\right) \tau_{\mathrm{p}}+B\left(\varrho_{\mathrm{n}}, \varrho_{\mathrm{p}}\right) \tau_{\mathrm{n}}+ \\
& +C\left(\varrho_{\mathrm{p}}, \varrho_{\mathrm{n}}\right)\left|\nabla_{\varrho_{\mathrm{p}}}\right|^{2}+C\left(\varrho_{\mathrm{n}}, \varrho_{\mathrm{p}}\right)\left|\nabla_{\varrho_{\mathrm{n}}}\right|^{2}+D\left(\varrho_{\mathrm{n}}, \varrho_{\mathrm{p}}\right) \nabla_{\varrho_{\mathrm{n}}} \cdot \nabla_{\varrho_{\mathrm{p}}},
\end{aligned}
$$

where the functions $A, B, C$, and $D$ are specified integrals involving the effective interaction at the local neutron and proton density, $\varrho_{\mathrm{SL}}\left(r k_{\mathrm{F}}\right)$ and $g\left(r k_{\mathrm{F}}\right)$.

One useful conceptual feature of this expansion is the fact that $H(\mathbf{R})$ may be separated into a term which is precisely the nucleon matter potential energy per particle, the kinetic energy, and terms which depend only on integrals of the long range part of $v_{\text {eff }}$ times $\tau$ and gradients of $\varrho$. The long range part of $v_{\text {eff }}$ is very close to the bare potential, which in turn is the most unambiguously determined part of the nuclear potential. Thus, the finite nucleus corrections are virtually independent of the

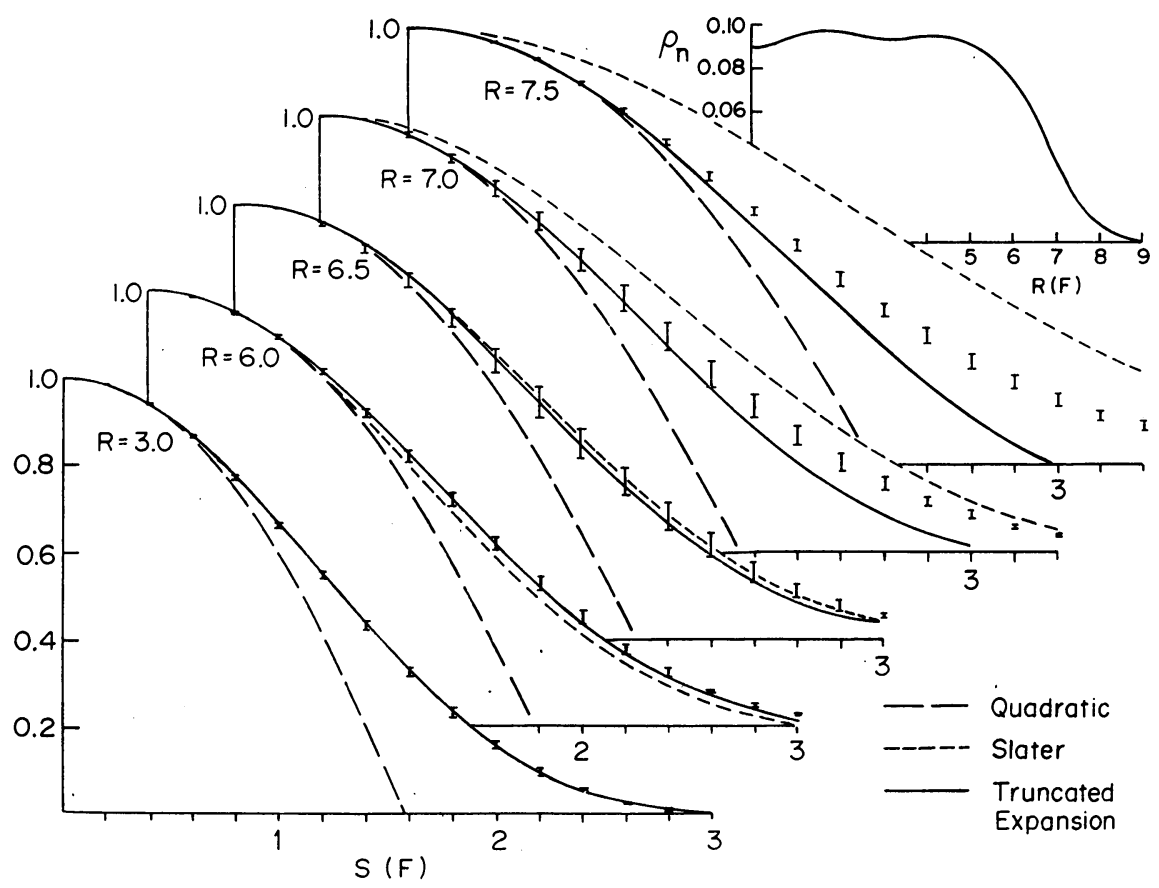

Fig. 7. Comparison of the square of the exact neutron density matrix in ${ }^{208} \mathrm{~Pb}$ with the truncated expansion, taken from Negele and Vautherin (1972). 
model of the nuclear force, and all ambiguities concerning the short range repulsion, off energy shell behavior, and higher order corrections enter only through the nuclear matter energy surface as a function of $\varrho_{n}$ and $\varrho_{p}$ as graphed in Figure 2.

Technically, the DME is much simpler than the DDHF theory, involving only a local Schrödinger equation with a position dependent effective mass. The results of this simplified theory have been compared in detail with the DDHF results (Negele and Vautherin, 1972) and are in excellent agreement. Because of the computational simplicity of the DME, it was possible to search more extensively over the parameters of the higher order correction adjustment and thereby obtain binding energies per particle which agree with experimental values within $0.1 \mathrm{MeV}$. The only additional change required to apply this theory of finite nuclei to the free neutron regime was to introduce a more careful parameterization of $A\left(\varrho_{n}, \varrho_{p}\right)$ in the region of very low neutron density so as to accurately reproduce the uniform nuclear matter calculations of Siemens and Pandharipande (1971).

TABLE I

$B E / A$ for pre-drip nuclei

\begin{tabular}{lll}
\hline Nucleus & Extrapolated value (MeV) & DME (MeV) \\
\hline${ }^{40^{122} \mathrm{Zr}}$ & 7.67 & 7.54 \\
${ }^{3^{120} \mathrm{Sr}}$ & 7.45 & 7.32 \\
${ }^{36^{118} \mathrm{Kr}}$ & 7.20 & 7.05 \\
\hline
\end{tabular}

Although our primary emphasis is on the free neutron regime, the DME theory of finite nuclei has been used to check the extrapolation by Myers and Swiatecki (1965) of the last pre-drip sequence of nuclei.

As shown in Table I, the DME results are in good agreement with the extrapolated values, and in particular, the differences between neighboring nuclei are in excellent agreement. Thus, we corroborate the results of Baym et al. (1971a) for the composition of matter prior to neutron drip. The spatial distribution of protons in ${ }^{118} \mathrm{Kr}$ is shown in Figure 11 for subsequent reference.

\section{The Neutron Drip Regime}

The equation of state in the neutron drip regime is obtained by minimizing the energy per nucleon of spherically symmetric configurations of nucleons in a Wigner-Seitz unit cell.

For charge neutrality, a cell with $\mathrm{Z}$ protons contains an equal number of electrons. Because of the large Fermi-Thomas screening length,

$$
1 / k_{\mathrm{FT}}=\left(\frac{4}{\pi} \frac{e^{2}}{\hbar c}\right) \frac{1}{k_{\mathrm{e}}},
$$

where $k_{\mathrm{e}}$ is the electron Fermi wave number, the electrons are approximated by a 
uniform gas, and the small screening correction is neglected (BBP). The kinetic energy per electron is then

$$
\frac{T}{Z}=m_{\mathrm{e}} c^{2}\left\{\frac{3}{8 x^{3}}\left[x\left(1+2 x^{2}\right)\left(1+x^{2}\right)^{1 / 2}-\ln \left(x+\left(1+x^{2}\right)^{1 / 2}\right)\right]-1\right\},
$$

where $x=\hbar k_{\mathrm{e}} / m_{\mathrm{e}} c$. The Coulomb exchange energy for the electrons is of the order of $e^{2} / \hbar c$ times the mean electron kinetic energy and is also neglected. With these approximations, the electron chemical potential is

$$
\begin{aligned}
\mu_{\mathrm{e}}=\left(k_{\mathrm{e}}^{2} \hbar^{2} c^{2}+m_{\mathrm{e}}^{2} c^{4}\right)^{1 / 2}- & m_{\mathrm{e}} c^{2}+\hbar c \times \\
& \times \int \mathrm{d}^{3} r_{1} \mathrm{~d}^{3} r_{2}\left[\varrho_{\mathrm{p}}\left(r_{1}\right)+\varrho_{\mathrm{e}}\left(r_{1}\right)\right]_{\left|r_{12}\right|}^{e^{2}} \varrho_{\mathrm{e}}\left(r_{2}\right) .
\end{aligned}
$$

The nuclear energy for the unit cell is expressed in terms of the nucleon wave functions using the DME Hamiltonian density functional. The direct Coulomb energy is calculated straightforwardly from the electron and proton densities, and the Slater

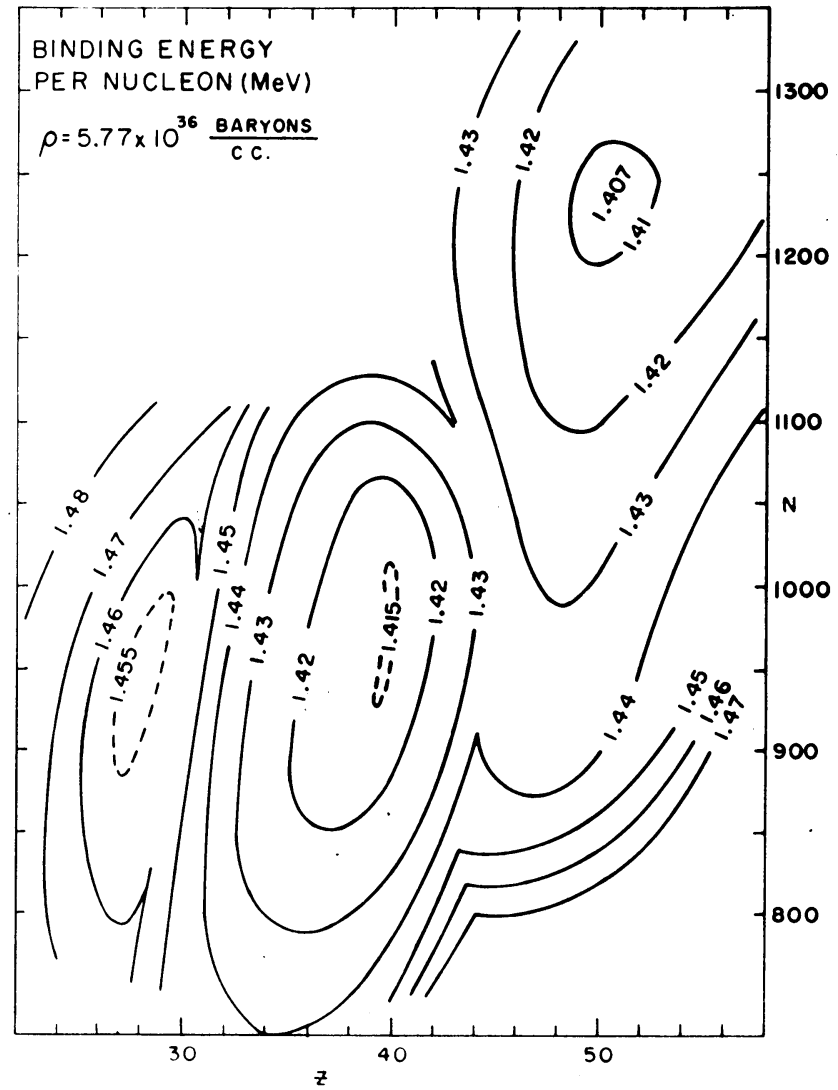

Fig. 8. Contour plot of energy per particle as a function of number of protons and neutrons in a unit cell. 


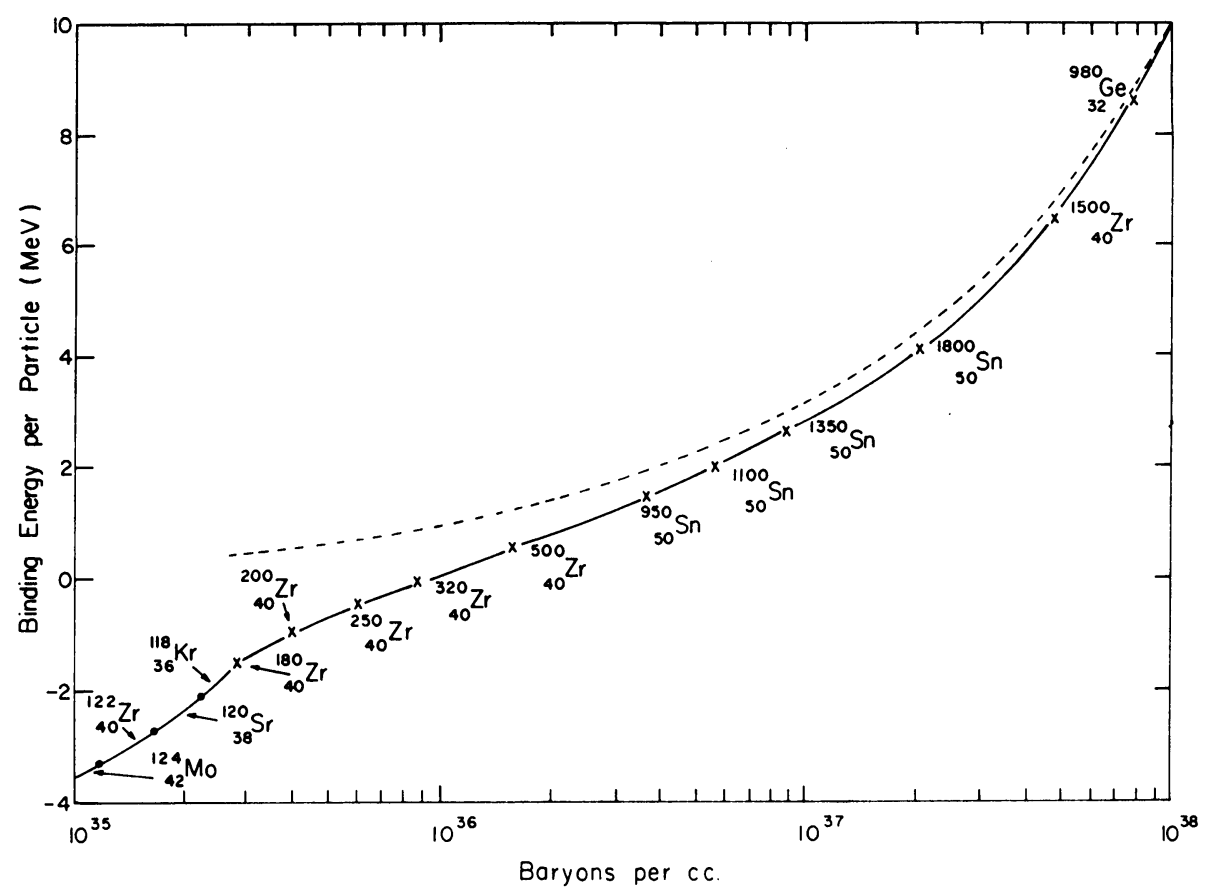

Fig. 9. Energy per particle versus baryon density.
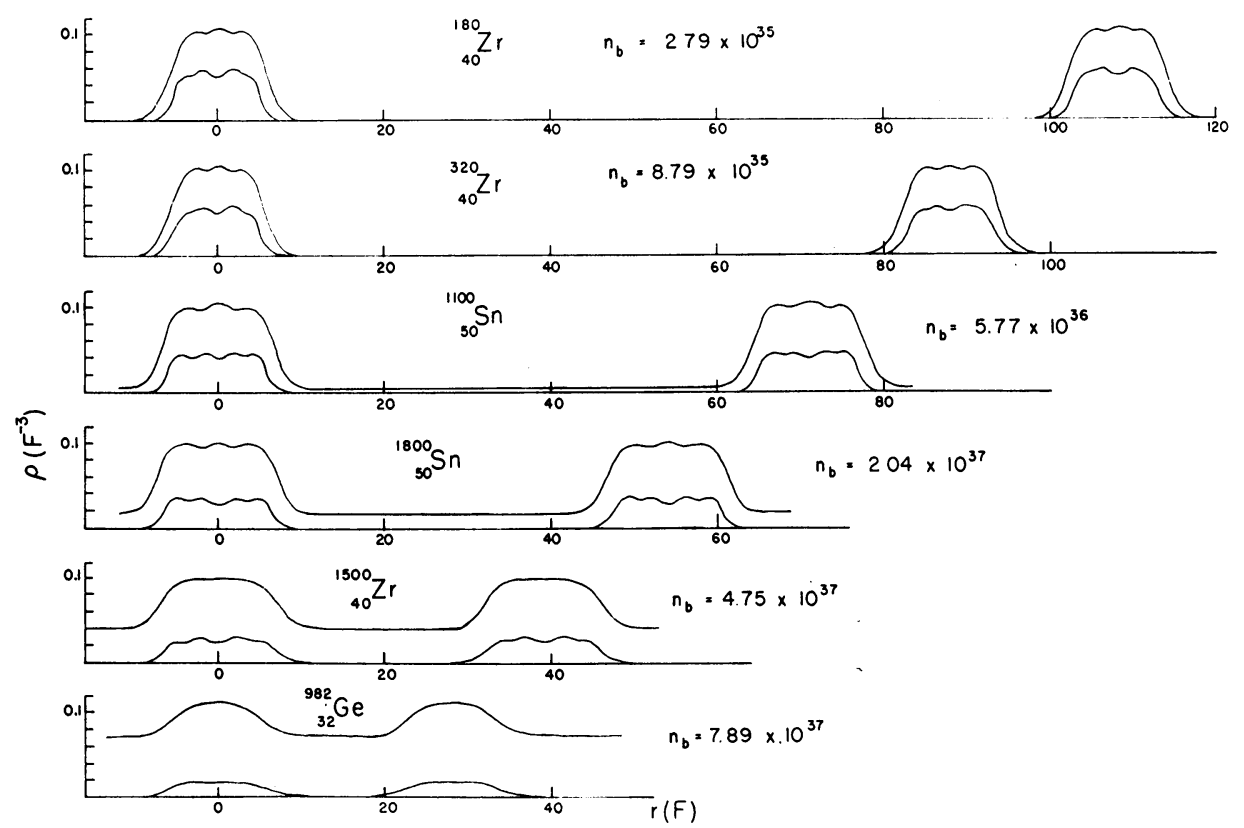

Fig. 10. Proton and neutron density distributions occurring along an axis joining the centers of two adjacent unit cells. 
approximation is used for the nuclear exchange Coulomb energy. Since the total energy expression is variational in the nuclear wave functions, the proton and neutron chemical potentials, $\mu_{\mathrm{P}}$ and $\mu_{\mathrm{N}}$, are given by the eigenvalues of the last occupied orbitals.

In addition to the approximations discussed previously in connection with the DME,

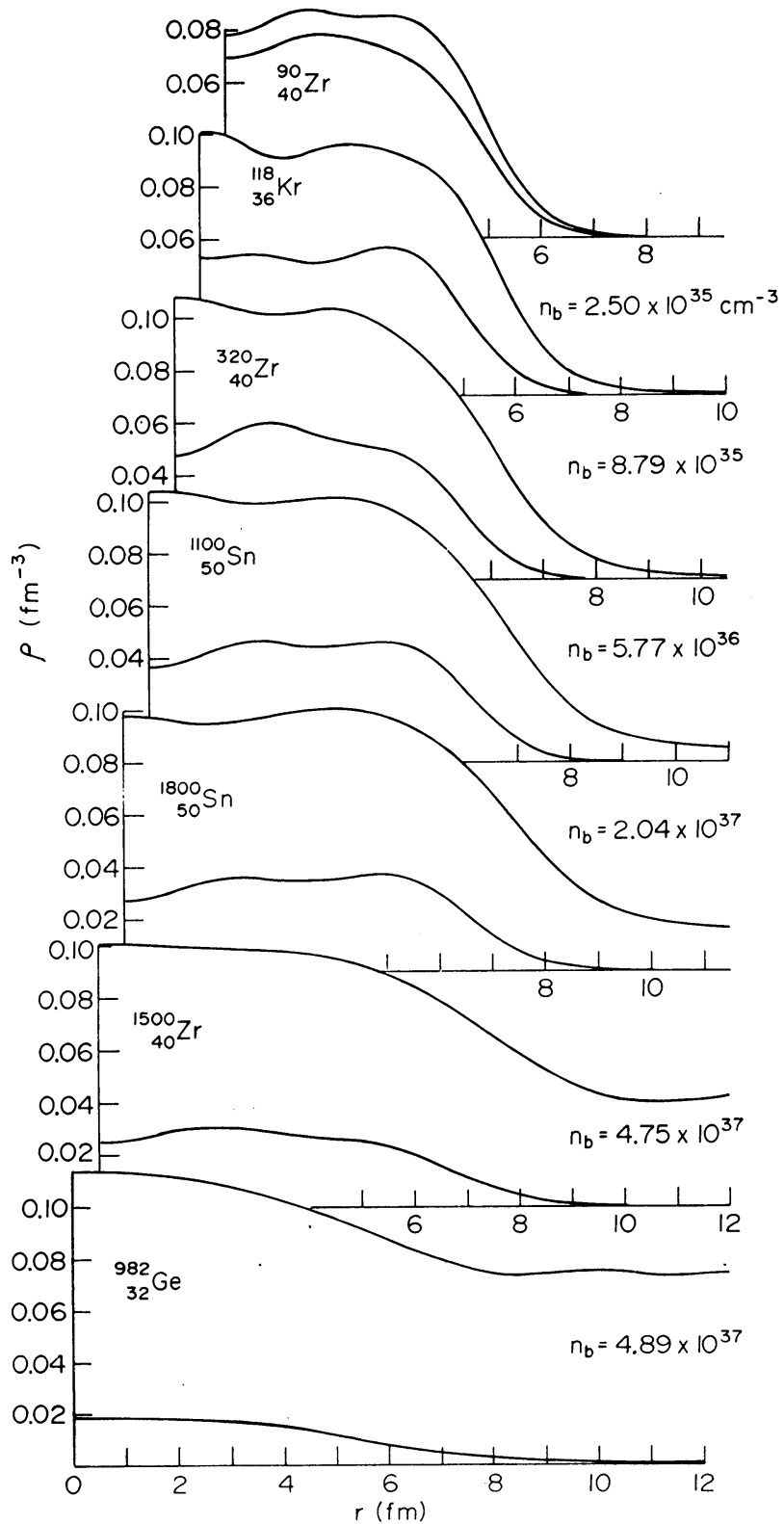

Fig. 11. Proton and neutron densities versus distance from the center of a unit cell. 


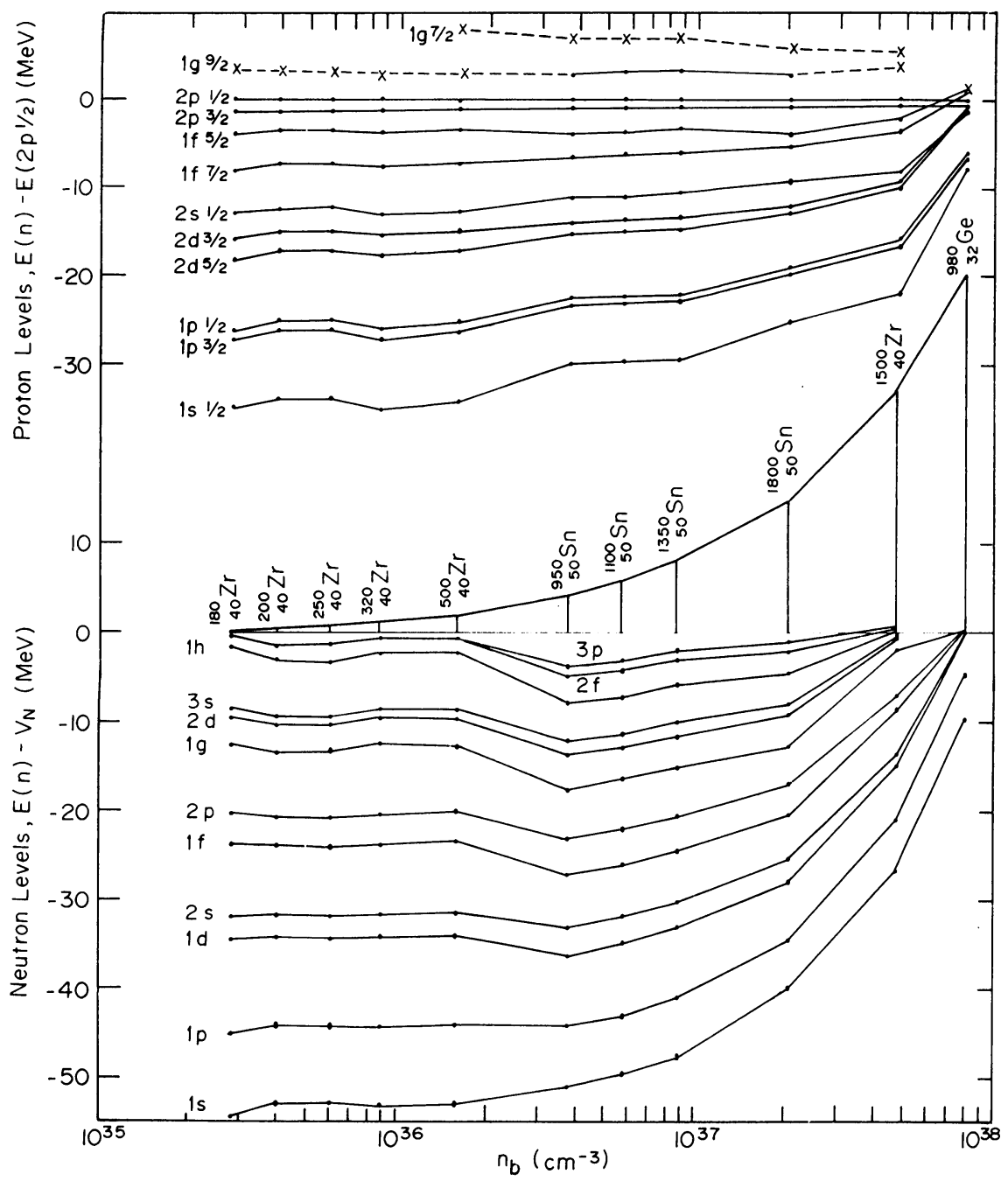

Fig. 12. Single particle spectrum of protons and neutrons.

it is extremely useful to omit the spin orbit splittings for neutron states, thereby reducing the total number of wave functions to be considered by almost one half. Since the one-body spin orbit potential is proportional to $(1 / r)(\mathrm{d} \varrho / \mathrm{d} r)$ and the spectrum of the continuum neutron states is essentially determined by the uniform neutron gas region, the effect of the spin orbit force on the neutron level ordering is negligible. Thus the density and energy density obtained by filling the barycentric states representing the average of the levels $j=l-\frac{1}{2}$ and $j=l+\frac{1}{2}$ with $2 l+1$ particles should be an excellent approximation. For protons, however, the spin orbit splitting is crucial in determining the level ordering and thus which orbitals are actually occupied. In order to retain a theory which is completely variational, the appropriate proton spin 
orbit term to be added to the energy density is (Negele and Vautherin, 1972) $H_{\mathrm{SO}}=W / 2\left[\nabla\left(\varrho_{\mathrm{N}}+2 \varrho_{\mathrm{P}}\right) \cdot \mathbf{J}_{\mathrm{P}}\right]$ where $\mathbf{J}_{\mathrm{P}}$ is the proton spin density $\mathbf{J}_{\mathrm{P}}=\left(\mathbf{r} / r^{4}\right) \sum_{\mathrm{i}} j_{\mathrm{i}}\left[\left(j_{\mathrm{i}}+\right.\right.$ $\left.+1)-l_{\mathrm{i}}\left(l_{\mathrm{i}}+1\right)-\frac{3}{4}\right] u_{\mathrm{i}}^{2}(r)$ and $W$ is a constant determined from the two-body spin orbit force.

With the approximations specified above, it is straightforward to perform a selfconsistent Hartree-Fock calculation for the nuclear wave functions in a unit cell, for a given cell radius and number of neutrons and protons. An initial guess is made for the single particle potentials and effective masses and the radial wave functions and eigenvalues are calculated subject to the boundary condition that the wave function or its derivative must vanish at the cell radius, depending on parity. The lowest $N$ neutron states and $Z$ proton states are occupied, $\varrho$ and $\tau$ are calculated for neutrons and protons and new potentials and effective masses are obtained. The lowest $N$ and $Z$ states for the new potentials are then filled and the process is iterated until self-consistency is achieved. Because of the multiplicity of local minima, it is necessary to try several dissimilar initial guesses for the potentials to assure that the absolute minimum energy configuration has been obtained.

To determine the minimum binding energy per nucleon at a specified baryon density,

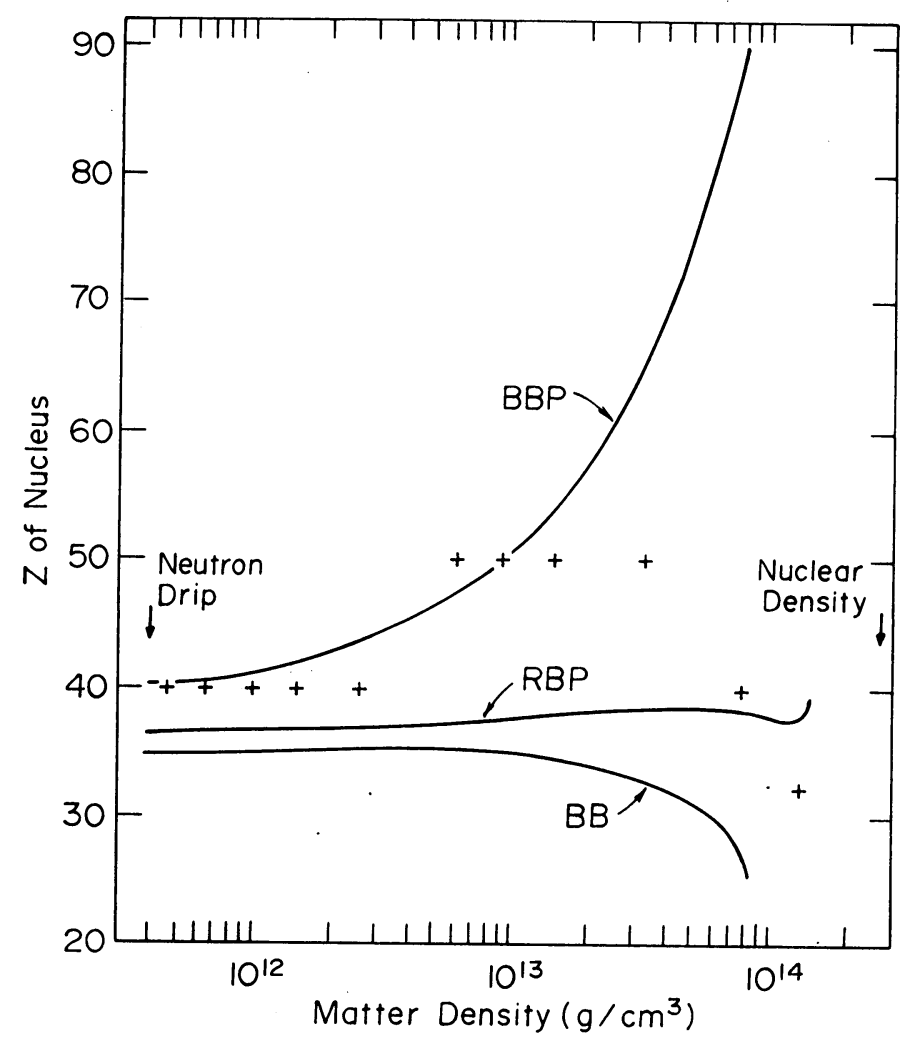

Fig. 13. Number of protons per nucleus, denoted by crosses, compared with previous predictions taken from Ravenhall et al. (1972). 
TABLE II

Numerical results in the free neutron regime. - The quantities $\tilde{Q}_{\mathrm{G}}$ and $\tilde{x}$ are the approximate density of the exterior neutron gas and the approximate ratio of protons to neutrons in nuclei respectively, and cannot be defined uniquely due to density fluctuations. All other quantities are defined in the text.

\begin{tabular}{|c|c|c|c|c|c|c|c|c|}
\hline $\begin{array}{l}n_{\mathrm{b}} \\
\left(\mathrm{cm}^{-3}\right)\end{array}$ & $N$ & $Z$ & $\begin{array}{l}\mu_{\mathrm{N}} \\
(\mathrm{MeV})\end{array}$ & $\begin{array}{l}\mu_{\mathrm{P}} \\
(\mathrm{MeV})\end{array}$ & $\begin{array}{l}\tilde{\varrho}_{\mathrm{G}} \\
\left(\mathrm{fm}^{-3}\right)\end{array}$ & $\tilde{x}$ & $\frac{E}{A}-m_{\mathrm{n}}$ & $\frac{E_{\mathrm{gas}}}{A}-m_{\mathrm{n}}$ \\
\hline & & & & & & & $(\mathrm{MeV})$ & $(\mathrm{MeV})$ \\
\hline $2.79 \times 10^{35}$ & 140 & 40 & 0.2 & -26.8 & $4 \times 10^{-5}$ & 0.53 & -1.425 & 0.436 \\
\hline $4.00 \times 10^{35}$ & 160 & 40 & 0.3 & -29.4 & $9.7 \times 10^{-5}$ & 0.53 & -0.962 & 0.543 \\
\hline $6.00 \times 10^{35}$ & 210 & 40 & 0.6 & -29.5 & $2.6 \times 10^{-4}$ & 0.53 & -0.462 & 0.692 \\
\hline $8.79 \times 10^{35}$ & 280 & 40 & 1.0 & -28.5 & $4.8 \times 10^{-4}$ & 0.53 & -0.050 & 0.865 \\
\hline $1.59 \times 10^{36}$ & 460 & 40 & 1.4 & -29.4 & $1.2 \times 10^{-3}$ & 0.52 & 0.541 & 1.214 \\
\hline $3.73 \times 10^{36}$ & 900 & 50 & 2.6 & -33.6 & $3.0 \times 10^{-3}$ & 0.46 & 1.465 & 1.926 \\
\hline $5.77 \times 10^{36}$ & 1050 & 50 & 3.3 & -34.5 & $4.7 \times 10^{-3}$ & 0.45 & 1.966 & 2.408 \\
\hline $8.91 \times 10^{36}$ & 1300 & 50 & 4.2 & -35.8 & $7.8 \times 10^{-3}$ & 0.44 & 2.610 & 2.981 \\
\hline $2.04 \times 10^{37}$ & 1750 & 50 & 6.5 & -43.6 & $1.84 \times 10^{-2}$ & 0.35 & 4.097 & 4.422 \\
\hline $4.75 \times 10^{37}$ & 1460 & 40 & 10.9 & -54.0 & $4.36 \times 10^{-2}$ & 0.28 & 6.428 & 6.660 \\
\hline $7.89 \times 10^{37}$ & 950 & 32 & 15.0 & -68.3 & $7.37 \times 10^{-2}$ & 0.16 & 8.611 & 8.657 \\
\hline
\end{tabular}

one must search over the number of nucleons in the unit cell, thus specifying the cell radius, and the ratio of neutrons to protons, as well as the spatial distribution of nucleons described above. The results of such a search for a preliminary version of this theory are shown as a contour plot in Figure 8. One notes several local minima generated by the energy fluctuations arising from the shell closures for 28,40 , and 50 protons, and in this case the absolute minimum occurs at $Z=50$. In practice, the search is greatly expedited by considering only $\beta$-stable configurations. At each iteration one computes $E(Z+1, N-1) \sim \mu_{\mathrm{e}}+\mu_{\mathrm{p}}-\mu_{\mathrm{n}}+m_{\mathrm{e}}+m_{\mathrm{p}}-m_{\mathrm{n}}$ and converts neutrons into protons plus electrons or vice-versa. The resulting $\beta$-stable self consistent solution corresponds to the minimum along a line of constant $N+Z$ in Figure 8, and one has only to perform a single parameter search over the number of particles in a cell.

The resulting ground state solutions for 11 densities are presented in Table II and Figure 9. Since it is impossible to distinguish neutrons in nuclei from those in the gas, we adopt the unusual convention of labeling nuclei by the total number of nucleons in the unit cell. In Figure 9, the energies per particle for the ground state configurations, denoted by the crosses, are compared with the energies of a $\beta$-stable uniform gas of electrons, protons, and neutrons, thus demonstrating the significant gain in binding energy obtained by forming a Coulomb lattice of nuclei surrounded by a low density neutron gas.

The spatial distribution of neutrons and protons at various densities is presented in Figures 10 and 11. Figure 10 shows the densities obtained along a line joining the centers of two adjacent unit cells. As the baryon density increases, one observes a smooth, systematic progression of configurations. The cell radius decreases, the neutron gas density increases and the density of protons in nuclei decreases. By the time one reaches ${ }^{982} \mathrm{Ge}$, the difference in energies of various local minima are sufficiently 
small that it is not meaningful to proceed to higher density. The energy per baryon is already very close to the uniform gas result, and it seems quite reasonable to assume that the density distributions at higher density continue the systematic behavior and smoothly approach a uniform density.

In Figure 11, the density distributions of the nuclei at the center of the unit cells are shown in greater detail. For comparison, ${ }^{90} \mathrm{Zr}$, a naturally occurring isotope, and ${ }^{118} \mathrm{Kr}$, the last pre-drip nucleus, are also presented. In addition to the systematic

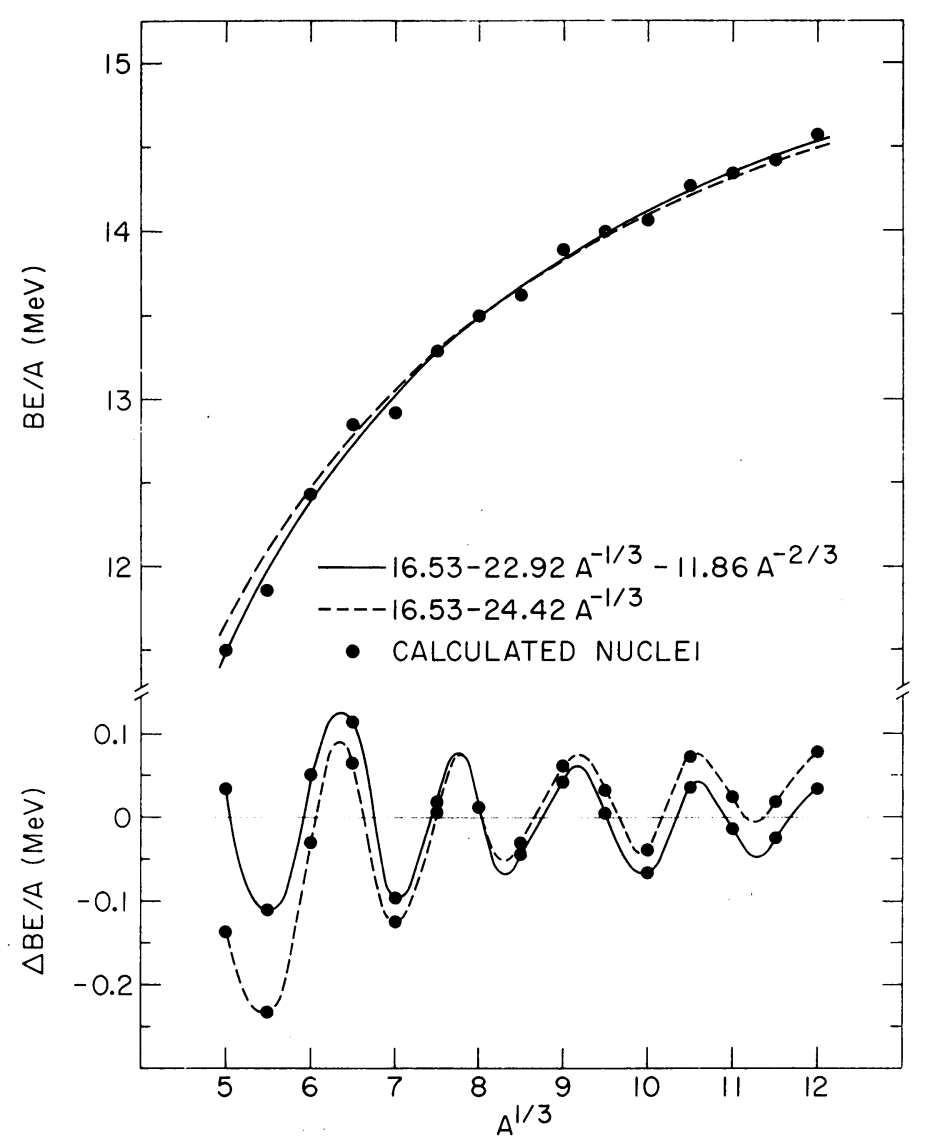

Fig. 14. Least squares fit to the masses of large, mirror nuclei to determine the curvature coefficient.

effects mentioned previously, one also observes a strong systematic increase of the nuclear surface thickness and the diminishing of neutron density fluctuations as the number of neutrons becomes sufficiently large and one approaches a statistical regime.

One of the most striking features of Figures 10 and 11 is the degree to which the nuclei in the free neutron regime resemble ordinary nuclei. This similarity is also manifested in the behavior of the single particle energies, as shown in Figure 12. In 
order to eliminate irrelevant constant shifts in the absolute single particle energies, relative energy spacings are plotted with the proton energies shown relative to the $2 p_{1 / 2}$ state and the neutron energies shown relative to the continuum. The fact that the usual shell model level sequence is maintained throughout the free neutron regime and that the interior nuclear density does not deviate significantly from the density of ordinary nuclei strongly suggest that the DDHF theory should be just as reliable in this regime as in observable nuclei where it has been experimentally tested.

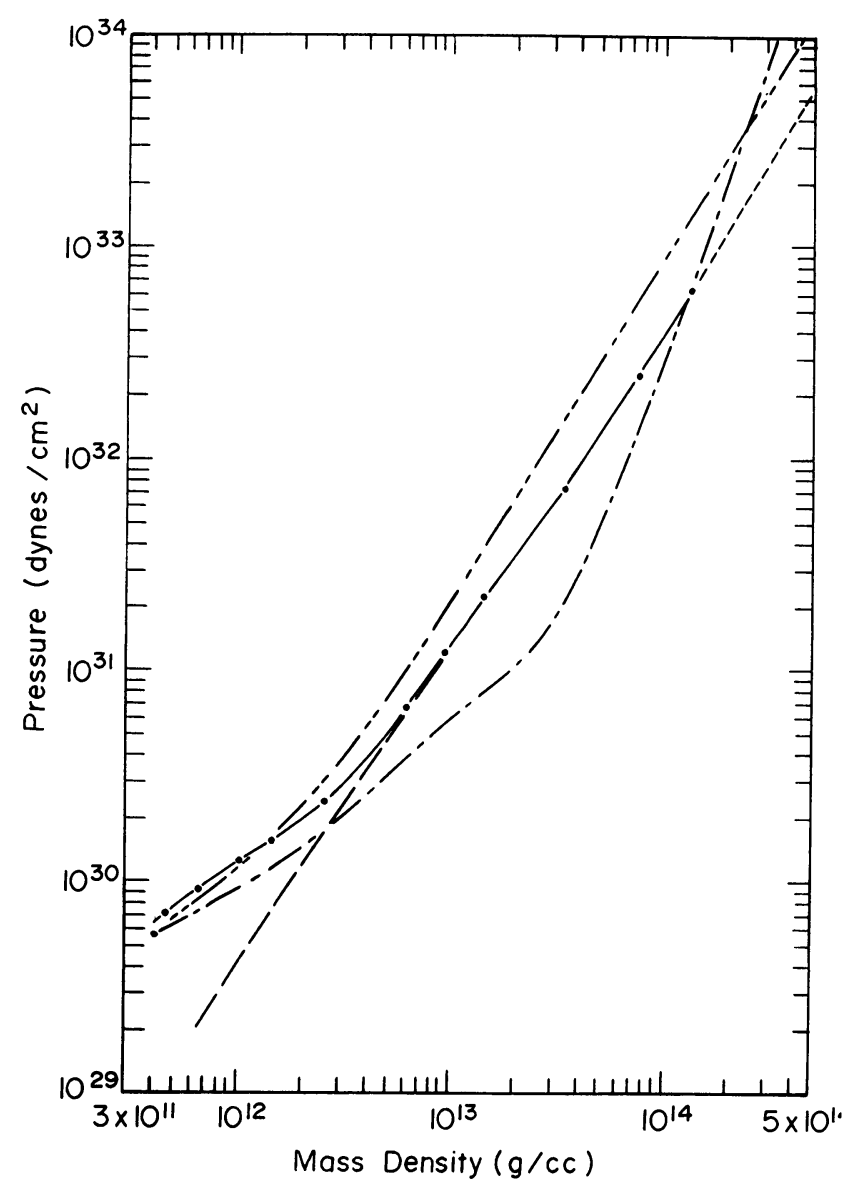

Fig. 15. Equation of state compared with previous predictions taken from BBP.

The composition of the resulting nuclei in this theory are compared with the predictions of BBP, Buchler and Barkat (1971a, b) (BB), and Ravenhall et al. (1972) (RBP) in Figure 13. We have already argued that RBP is the most reliable of the three previous theories. The equilibrium conditions derived by BBP show that if the nuclear surface energy is expressed in the form $B E \sim W_{\text {SURF }} A^{2 / 3}$, then the equilibrium size of the nucleus is given by $W_{\mathrm{SURF}} A^{2 / 3}=2 E_{\mathrm{COUL}}$, where $E_{\mathrm{COUL}}$ is the total Coulomb energy 


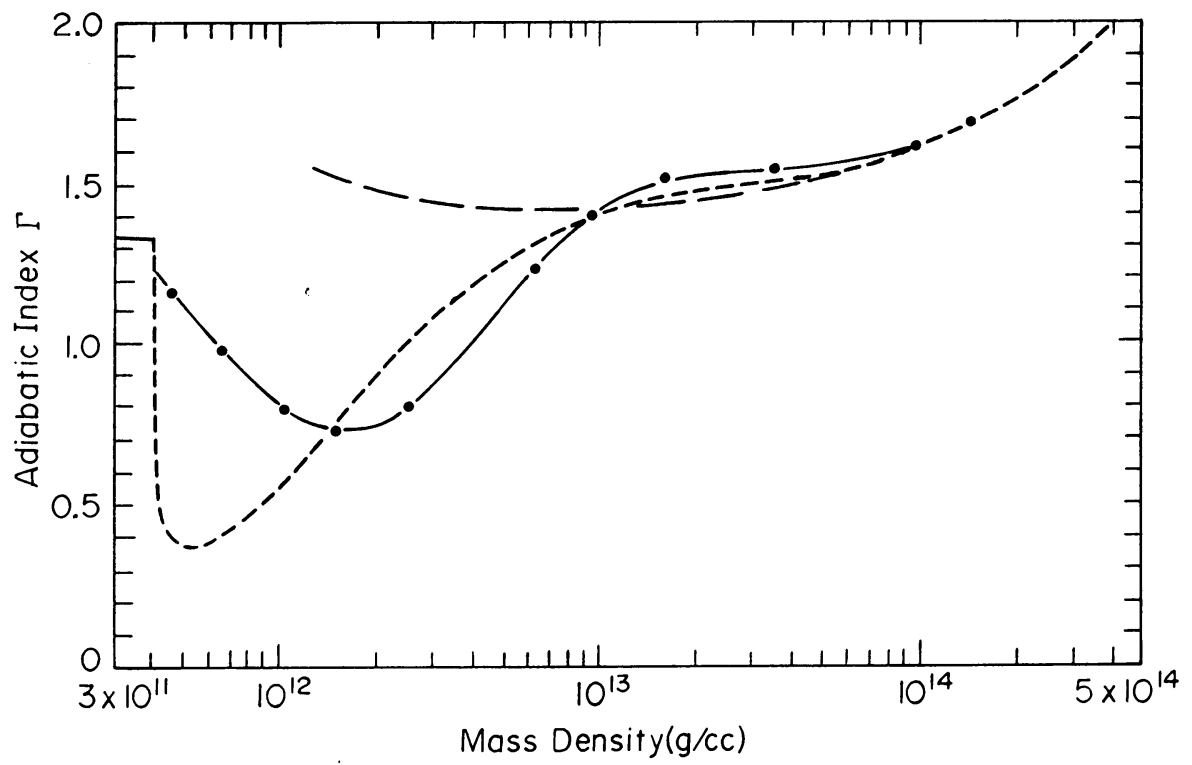

Fig. 16. Adiabatic index compared with the BBP result.

per nucleus, including the lattice energy. However, in the spherical geometry of a Wigner-Seitz unit cell, in contrast to a plane surface, there are additional contributions to the surface energy proportional to $A^{1 / 3}$ arising from the curvature of the surface as well as a small pressure term which we will ignore for the present argument. The curvature term may be evaluated by calculating a sequence of unphysically large, mirror nuclei with the Coulomb force turned off. The resulting binding energies are least squares fit with a mass formula of the form $B E=W_{\mathrm{VOL}} A+W_{\mathrm{SURF}} A^{2 / 3}+W_{\mathrm{CURV}} A^{1 / 3}$ as shown in Figure 14. If one repeats the argument of $\mathrm{BBP}$ with this expression, the new equilibrium condition is $\left(W_{\mathrm{SURF}}+2 W_{\mathrm{CURV}} A^{-1 / 3}\right) A^{2 / 3}=2 E_{\mathrm{COUL}}$. Thus, substituting the values from Figure 14, although the curvature term in the binding energy is only about $11 \%$ of the surface term for $A \sim 200$, it shifts the equilibrium size by $22 \%$. Taking an average value of $Z$ from RBP to be 37, this effect would be expected to shift the

TABLE III

Coefficients for the equation of state

\begin{tabular}{lll}
\hline$I$ & Ground state $C_{I}$ & \multicolumn{1}{c}{ Uniform gas $C_{I}$} \\
\hline 0 & -4.0 & \multicolumn{1}{l}{} \\
1 & $2.8822899 \times 10^{-1}$ & \multicolumn{1}{l}{4.0} \\
2 & $5.9150523 \times 10^{-1}$ & $-4.0373482 \times 10^{-2}$ \\
3 & $9.0185940 \times 10^{-2}$ & $6.0455728 \times 10^{-2}$ \\
4 & $-1.1025614 \times 10^{-1}$ & $-1.5307639 \times 10^{-2}$ \\
5 & $2.9377479 \times 10^{-2}$ & $3.4774416 \times 10^{-3}$ \\
6 & $-3.2618465 \times 10^{-3}$ & $-4.3627154 \times 10^{-4}$ \\
7 & $1.3543555 \times 10^{-4}$ & $2.3383473 \times 10^{-5}$
\end{tabular}


charge to 45 . Because of shell effects, as demonstrated in Figure 8 , the equilibrium point in our present theory is shifted away from 45 , either to the shell closure at 40 or to the closure at 50 , but it is significant that the average shift is accurately explained by this curvature effect.

Given the total ground state energy per particle, including masses, $E_{\mathrm{T}}$, as a function of baryon density, $n_{\mathrm{b}}$, the pressure, adiabatic index and mass density are determined as follows: $P=n_{\mathrm{b}}^{2}\left(\partial E_{\mathrm{T}} / \partial n_{\mathrm{b}}\right), \Gamma=\left(n_{\mathrm{b}} / P\right)\left(\partial P / \partial n_{\mathrm{b}}\right)$ and $\varrho=n_{\mathrm{b}} E_{\mathrm{T}} / c^{2}$. Since searches were carried out only at 11 discrete densities, it was necessary to curve fit the resulting energies shown in Figure 9 with a smooth function. The total energies per baryon, $E_{\mathrm{T}}$, for both the ground state and uniform gas configurations were fit by the following function:

$$
E_{\mathrm{T}}=m_{\mathrm{n}}+c_{0}+\exp \left\{\sum_{I=1}^{7} c_{I} x^{(I-1)}\right\},
$$

where $E_{\mathrm{T}}$ is in $\mathrm{MeV}, m_{\mathrm{n}}$ is the neutron mass, $x=\ln \left(n_{\mathrm{b}} \times 10^{-35}\right), n_{\mathrm{b}}$ is the baryon density in baryons per $\mathrm{cm}^{3}$, and $c_{0}$ through $c_{7}$ are tabulated in Table III.

The equation of state obtained by fitting the 11 ground state nuclear configurations in the free neutron regime is shown in Figure 15 by the solid line. At high density, it joins smoothly onto the BBP curve, denoted by the short dashed line. The equation of state for a uniform gas is indicated by the long dashed curve, and becomes indistinguishable from the ground state curve at intermediate densities. For comparison, the Harrison-Wheeler equation of state (Hartle and Thorne, 1968) and the $V_{\gamma}$ equation of state of Langer et al. (1969) are also plotted as taken from BBP.

The adiabatic index calculated with the present theory is shown in Figure 16 by the solid line. One should note that taking the second derivative of the curve fit to the 11 calculated energies strongly amplifies noise in the fit, and therefore undue significance should not be attached to the fine details of this curve. For comparison, the adiabatic index obtained for a uniform gas is shown by the long dashes and the BBP result is shown by the short dashes.

\section{Conclusion}

We have attempted to show in this work that it is possible to construct a reliable theory of a nucleon many-body system derived from the two-body nucleon-nucleon interaction. The relevant two-body correlations are incorporated in a two-body effective interaction, and the energy density is expressed as an extremely simple functional of the density and kinetic energy density via the density matrix expansion. This theory yields excellent agreement with experimentally observable properties of finite nuclei and should provide a reliable extrapolation to the nucleus-like clusters occurring in the free neutron regime of dense matter.

The primary uncertainty affecting the equation of state we have calculated in the free neutron regime is the small uncertainty in the function specifying the uniform matter potential energy for a small ratio of protons to neutrons. The adjustment for the higher order corrections, the ambiguity in off-energy-shell behavior of the nuclear force, and the omission of pairing correlations (Yang and Clark, 1971), all contribute 
to the uncertainty in the energy of a neutron gas. Fortunately, throughout most of the free neutron regime, the nuclear clusters occurring in the center of the unit cell are insensitive to small changes in the energy functional for a neutron gas, so that the composition and energy of the cluster is essentially unaffected. Since most of the baryons in the cell are neutrons in the gas, their energy change may be computed directly from the change in the neutron gas energy and thus the change in the total energy and therefore the'equation of state may be obtained from the data in Table II without repeating the lengthy self-consistent calculations reported in this work.

Finally, the substantial agreement between the final equation of state obtained in this work and that of BBP suggests that calculations of Baym et al. (1971a) based on the BBP results should be essentially unchanged by the present work.

\section{Acknowledgments}

It is a pleasure to acknowledge the contribution of $\mathrm{D}$. Vautherin who collaborated in the development of the density matrix expansion and in the writing of the program to calculate wave functions in a Wigner Seitz cell. In addition, this work has benefitted greatly at various stages from discussions with $\mathrm{H}$. A. Bethe, G. Baym and C. J. Pethick. The hospitality of the Niels Bohr Institute during the spring of 1970, where the author's interest in this problem was first stimulated, and of Brookhaven National Laboratory during the summer of 1972, where this manuscript was prepared, is gratefully acknowledged. This work was supported in part through funds provided by the U.S. Atomic Energy Commission under Contract No. AT(11-1)-3069.

\section{References}

Arponen, J.: 1971, University of Helsinki (preprint).

Barkat, Z., Buchler, J. R., and Ingber, L.: 1972 (to be published).

Baym, G. A., Pethick, C., and Sutherland, P.: 1971a, Astrophys. J. 170, 299.

Baym, G. A., Bethe, H. A., and Pethick, C. J.: 1971b, Nucl. Phys. A175, 225.

Bethe, H. A.: 1971, Ann. Rev. Nucl. Sci. 21, 93.

Bethe, H. A., Börner, G., and Sato, K.: 1970, Astron. Astrophys. 7, 279.

Brown, G. E. and Green, A. M.: 1969, Nucl. Phys. A173, 1.

Buchler, J. R. and Barkat, Z.: 1971a, Phys. Rev. Letters 27, 48.

Buchler, J. R. and Barkat, Z.: 1971b, Astrophys. Letters 7, 167.

Campi, X. and Sprung, D. W.: 1972, Nucl. Phys. A194, 401.

Hartle, J. B. and Thorne, K. S.: 1968, Astrophys. J. 153, 807.

Langer, W.D., Rosen, L.C., Cohen, J. M., and Cameron, A. G. W.: 1969, Astrophys. Space Sci. 5, 259.

Myers, W. D. and Swiatecki, W. J.: 1965, UCRL Report 11980.

Negele, J. W.: 1970, Phys. Rev. C1, 1260.

Negele, J. W. and Vautherin, D.: 1972, Phys. Rev. C5, 1472.

Pines, D.: 1970, Proc. of XVII Internationàl Conf. on Low Temperature Physics.

Ravenhall, D. G., Bennett, C. D., and Pethick, C. J.: 1972, Phys. Rev. Letters 28, 978.

Reid, R. V.: 1968, Ann. Phys. N.Y. 50, 411.

Siemens, P. J.: 1970, Nucl. Phys. A141, 225.

Siemens, P. J. and Pandharipande, V. R.: 1971, Nucl. Phys. A173, 561.

Skyrme, T. H. R.: 1959, Nucl. Phys. 9, 615.

Sprung, D. W. L.: 1972, Advances in Nuclear Physics (to be published).

Vautherin, D. and Brink, D. M.: 1972, Phys. Rev. C5, 626.

Yang, C. H. and Clark, J. W.: 1971, Nucl. Phys. A174, 49. 